\title{
Superalimento para un mundo en crisis: Spirulina a bajo costo
}

\author{
Superfood for a world in crisis: Spirulina at low cost
}

Ernesto Ponce López $z^{1}$

\begin{abstract}
RESUMEN
Introducción: La Spirulina es una microalga que contiene un alto contenido proteico, ácidos grasos esenciales, minerales y vitaminas. Es un cultivo ideal para zonas desérticas y más aún donde el agua es alcalina. Es fácil de multiplicar y económica de producir. Se considera un superalimento. Es consumida por atletas de nivel olímpico y es el alimento de los astronautas. Sesenta gramos bastan para alimentar a una persona por día, con 10 gramos diarios se puede sobrevivir. El objetivo de este trabajo es dar la información necesaria para que pueda cultivarse en zonas rurales, como un complemento alimenticio. Material y Método: Se trabajó con la cepa Spirulina Platensis, en una planta piloto cerca de Arica, Norte de Chile, en sistema de estanques abiertos, en una instalación tipo invernadero, con agitadores electromecánicos de baja potencia. Resultados y conclusiones: Las respuestas indicaron medias de producción diarias desde $3 \mathrm{~g} / \mathrm{m}^{2}$ en otoño-invierno hasta $4 \mathrm{~g} / \mathrm{m}^{2}$ en primavera-verano, con un costo de producción bajo los US\$7/kg, siendo la mitad de lo que cuesta la carne de res de la calidad más alta. Se concluye que es recomendable su cultivo para pobladores de zonas rurales.
\end{abstract}

Palabras clave: Spirulina, acuacultura, alimentación.

\begin{abstract}
Introduction: Spirulina is a microalgae that contains a high protein, essential fatty acids, minerals and vitamins. It is an ideal culture for desert and even where water is alkaline Multiplication is easy and economical to produce. It is considered a superfood. Is consumed by Olympic level athletes and is the food of the astronauts. Sixty grams are enough to feed one person per day, with 10 grams daily can survive. The aim of this paper is to give the information to be cultivated in rural areas, as a food supplement. Material and Methods: We worked with the strain Spirulina Platensis, in a pilot plant near Arica, North of Chile, in open pond system in a greenhouse facility, with low power electromechanical shakers. Results and conclusions: The responses showed average daily production from $3 \mathrm{~g} / \mathrm{m}^{2}$ in autumn-winter to $4 \mathrm{~g} / \mathrm{m}^{2}$ in spring-summer, with a production cost below US\$ 7/kg, half of the cost of beef from the highest quality. We conclude that cultivation is recommended for residents of rural areas.
\end{abstract}

Key words: Spirulina, aquaculture, food.

\section{Introducción}

El consumo de microalgas azuladas Spirulina se inicia en el pasado en diferentes lugares del globo. Según las crónicas de los conquistadores españoles, los antiguos aztecas las recolectaban en la superficie del lago de Tenochtitlán, luego eran secadas y comercializadas en el mercado de la ciudad. A los corredores aztecas, portadores de pescado fresco desde la costa hasta los palacios de la nobleza, se les alimentaba con Spirulina. La distancia a recorrer era superior a $300 \mathrm{~km}$, por lo que el trayecto se completaba con postas donde los atletas indígenas corrían con una resistencia extraordinaria. En determinadas épocas del año eran recolectadas en canoas usando finas redes, secadas al sol y convertidas en tortillas, que sabían a queso. Complementaban la alimentación de frejoles, cebollas, ají y maíz. Durante la colonia las ordenanzas españolas hicieron olvidar estas algas. Cabe mencionar que también en el lago Chad, en África, los pobladores se alimentaban desde hacía siglos con Spirulina secada al sol y forma de galletas. Los habitantes de las riberas son sanos, atléticos, altos y buenos corredores.

Esta microalga existe en el planeta desde unos tres mil quinientos millones de años antes del presente. Las variedades más conocidas son la Spirulina Máxima y la Spirulina Platensis. El alga se divide en dos cada 7 horas, en condiciones ideales teóricamente puede generar unos $15.000 \mathrm{~kg} / \mathrm{ha}$ anuales de material seco, pero con tecnología apropiada los

1 Escuela Universitaria de Ingeniería Mecánica. Universidad de Tarapacá. Arica, Chile. E-mail: eponce60@gmail.com

Fecha de Recepción: 03 Octubre, 2012.

Fecha de Aceptación: 14 Enero, 2013. 
rendimientos mejoran. La Spirulina crece en medios adversos por lo que ha sido capaz de absorber los elementos necesarios para sobrevivir, siendo una de las primeras algas en realizar fotosíntesis, tiene una enorme capacidad de almacenar nutrientes. Su geometría es de espiral, sus células cilíndricas miden de 3 a 13 micras de diámetro y su largo puede tener entre 100 y 200 micras.

En el último tercio del siglo XX, en una planta industrial de extracción de soda en México, se buscaba una solución para eliminar "la contaminación" por algas en la laguna, lo que dio origen al redescubrimiento de las excelentes propiedades del "contaminante" que condujo a su explotación $\mathrm{y}$ al inicio de cientos de investigaciones sobre ella.

Es un cultivo ideal para zonas desérticas y más aún donde el agua salina no es adecuada para la agricultura convencional. Como crece en medios acuosos alcalinos y salobres, presenta pocas posibilidades de contaminarse. Su principal empleo es para alimentar personas y animales.

La Spirulina tiene cualidades inmunológicas, antioxidantes, antiviral, protector contra el cáncer, retiene metales pesados por lo que es un antitóxico. Se ha demostrado que es un regulador contra la hiperglicemia y la hiperlipidemia (Belay, 2002).

Está autorizada legalmente como complemento alimenticio en EE.UU., Europa, Japón y la Cuenca del Pacífico. En Estados Unidos la FDA (Departamento para la alimentación y medicamentos) la autorizó a partir de 1981, indicando que es una fuente proteica que contiene además vitaminas y minerales.

Los complementos sintéticos de vitaminas y minerales suelen no ser reconocidos por el organismo, al faltar los agentes capaces de fijar los iones metálicos y no-metálicos. Con la Spirulina la absorción es muy eficiente por parte del sistema digestivo, entre 85 y 95\% (Henrikson, 1994). Esto guarda relación con el sentido de giro de su espiral, compatible con el giro del ADN humano (efecto de chiralidad, que se empieza a usar en medicamentos de tecnología avanzada).

Es rica en vitamina $\mathrm{E}$ (agente de antienvejecimiento celular) y vitamina B1 (tiamina), importante para producir acetilcolina (un neurotransmisor). $\mathrm{La}$ vitamina B12 es necesaria para un normal funcionamiento del tejido nervioso, ayudando a mantener la vaina de mielina que rodea a los axones neuronales. La microalga aporta dosis de hierro, zinc, cobre y germanio, siendo este último un semiconductor que conserva los contactos eléctricos a través del sistema neurológico. La Spirulina contiene 65\% de proteínas y aminoácidos; 55 de lípidos, $7 \%$ de minerales; $20 \%$ de hidratos de carbono y $3 \%$ de humedad. Dentro de los aminoácidos esenciales esta microalga tiene: isoleucina $5,6 \%$; leucina $8,7 \%$; lisina $4,7 \%$, metionina $2,3 \%$; fenilalanina $4,5 \%$; treonina $5,2 \%$; triptófano $1,5 \%$ y valina $6,5 \%$. Las vitaminas aportadas por $10 \mathrm{~g}$ de Spirulina en función de la dieta diaria recomendada, son: vitamina A betacaroteno 4,60 veces; vitamina B1 tiamina 0,21 ; vitamina $\mathrm{B} 2$ riboflavina 0,21 ; vitamina $\mathrm{B} 3$ (niacina) 0,07 ; vitamina B6 (piridoxina) 0,04 ; vitamina B12 5,33 veces; vitamina $\mathrm{E}$ alfa-tocoferol 0,03 (Henrikson, 1994).

Con respecto al consumo de agua en litros por kilogramo producido y al porcentaje de proteína aportada, la Spirulina tiene un $65 \%$ de proteínas y consume 2.499 litros; la soja tiene $34 \%$ de proteínas consumiendo 8.860 litros; el maíz tiene $9 \%$ de proteínas y consume 12.416 litros; una res en establo alimentada con grano tiene $20 \%$ de proteínas y consume 104.000 litros (Earthrise Farms 1998).

Actualmente, en muchos países se extrae en forma tecnificada en grandes piscinas de cultivo. Los principales productores son EE.UU., Japón, India, China, entre otros. Estudios y producciones importantes se realizan en Rusia, Francia, Alemania, Israel, Italia y España, por mencionar algunos. En la actualidad los procesos de cultivo generalmente casi no están automatizados. Los parámetros se controlan en forma manual y según la experiencia de los operadores. En grandes instalaciones debe contarse con una automatización eficiente para controlar algunos parámetros críticos: nivel de $\mathrm{pH}$, densidad de población y temperatura. La ventaja de un control radica en adicionar la cantidad correcta de nutrientes en función de las necesidades reales; realizar la cosecha cuando la densidad de algas alcanza un nivel determinado (antes de llegar a la sobrepoblación y muerte súbita del cultivo) y reducir la temperatura cuando se alcanzan valores peligrosos en verano. La metodología que se emplea consiste en la instalación de sensores para controlar mediante un microprocesador la marcha del proceso. Este envía las señales correctivas a los actuadores: dosificador de nutrientes, bomba de cultivo y mecanismos para reducir la temperatura. Los resultados han demostrado que el proceso puede controlarse sin supervisión directa, ningún cultivo se ha perdido por el control automático y los tiempos de operación se reducen considerablemente, frente 
a los considerados clásicos (Ponce \& Ponce, 2008). Actualmente se dispone de un sistema inteligente diseñado y construido en la UTA para controlar el cultivo y la densidad poblacional (Ponce et al., 2008).

Los más modernos y eficientes sistemas son de fotobiorreactores tubulares, empleados en lugares extremos como Siberia. Son compactos al ser cerrados y controlados en forma automática, necesitan poca mantención.

Por su gran resistencia se piensa que deberían ser las primeras algas en servir de alimento para colonias fuera del planeta, teniendo la ventaja de generar oxígeno en grandes cantidades y absorber $\mathrm{CO}_{2}$, tal como ocurrió en los comienzos de la vida en la Tierra.

\section{Metodología}

Se empleó el sistema más conocido y económico, que es el de estanque abierto de poca profundidad, $20 \mathrm{~cm}$, de forma rectangular con terminaciones de semicírculo en los extremos (Jourdan, 2002). Las superficies pueden variar desde 3 hasta $5.000 \mathrm{~m}^{2}$. El agua circula mediante agitadores de rueda con paletas. Es necesaria la agitación, para que todas las microalgas reciban igual cantidad de luz para la fotosíntesis y los nutrientes puedan mezclarse homogéneamente en el agua.

Se trabajó con dos estanques de $2,6 \mathrm{~m}^{2}$ de superficie cada uno y $0,2 \mathrm{~m}$ de profundidad. Estaban dentro de un invernadero de polietileno resistente a los rayos ultravioleta. Fue importante que estuviera debidamente cerrado, porque siendo una fuente alimenticia atrae a los insectos y animales. Los estanques fueron construidos en fibra de vidrio de $1,15 \times 2,5 \mathrm{~m}$, con una separación longitudinal en el centro para facilitar la circulación del agua (llamados "race way"). Se usaron agitadores de 1/4 HP, 4 paletas desplazaban el líquido a baja velocidad $(15 \mathrm{~cm} / \mathrm{s})$. El volumen de cada depósito fue de 420 litros.

En la segunda mitad del proyecto se trabajó con un estanque de mayor superficie, apoyado sobre un terreno liso y apisonado. Fue construido con una manga de polietileno negro para depósitos de agua, de espesor 0,1 $\mathrm{mm}$. Externamente estaba soportado por una estructura de listones de pino, el sistema más económico que puede montarse. También estaba bajo invernadero de plástico resistente a la luz ultravioleta.
Los valores de parámetros medios medidos fueron: temperatura 30 grados centígrados en el aire del invernadero y 27 grados en el agua de los estanques. Luminosidad de 1.800 lux en 12 horas de exposición diaria, a 18 grados de latitud. Los $\mathrm{pH}$ variaron de 8,85 a 9,76.

El agua empleada provenía de la red, debió ser dejada en reposo dos días para eliminar el cloro, antes de ponerse en contacto con las algas. Con la reposición en los estanques se tuvo el mismo cuidado.

El secado se realizó con un secador solar de superficie; $0,8 \mathrm{~m}^{2}$ en la tapa del portabandejas y $1 \mathrm{~m}^{2}$ en el colector conectado a su base.

Cultivo de la cepa: Se incrementó previamente la masa algar a partir de una muestra de $25 \mathrm{cc}$, duplicando el volumen cada día, empleándose primeramente botellas transparentes de policarbonato para continuar con depósitos de plástico de 20 litros de agua y nutrientes. Se trabajó con luz natural, en vez de tubos fluorescentes. Una vez obtenidos 60 litros, se vaciaron en el depósito tipo "race way", continúandose allí el proceso de duplicación hasta completar el volumen.

La solución usada para duplicar el cultivo fue:

Sal de mar: 18,2 gramos/litro;

$\mathrm{FeSO}_{4}$ : 0,01 gramos/litro;

$\mathrm{KH}_{2} \mathrm{PO}_{4}$ : 0,14 gramos/litro;

$\mathrm{KNO}_{3}: 4,4$ gramos/litro;

$\mathrm{NaHCO}_{3}:$ 6,0 gramos/litro.

Por seguridad y hasta el término del proyecto siempre se mantuvo botellas con cepas.

Alimentación con el sistema funcionando:

Sal de mar: 5 gramos/litro;

Sulfato férrico $\mathrm{FeSO}_{4}$ : 0,005 gramos/litro;

Fosfato de potasio $\mathrm{KH}_{2} \mathrm{PO}_{4}:$ 0,07 gramos/litro;

Salitre potásico $\mathrm{KNO}_{3}: 3,0$ gramos/litro;

Bicarbonato sódico NaHCO: 7,0 gramos/litro.

Los cambios deben realizarse gradualmente, no se debe olvidar que son seres vivos y pueden perecer por estrés, luego el suministro de nutrientes debe durar algunos minutos para no variar bruscamente la solución del estanque. Las algas deben ser alimentadas después de cada cosecha.

Controles: El pH debería mantenerse entre 9 y 11. Debe realizarse la cosecha antes de alcanzar el límite de $40 \mathrm{~g} / \mathrm{m}^{2}$ día, de lo contrario se corre el riesgo de perder todo el cultivo por sobrepoblación. Temperatura: en el agua de cultivo no debe sobrepasar de 30 grados Celsius. El funcionamiento del agitador era de 15 minutos en cada hora, a partir 
de las 9:00 hasta las 18:00 horas, el control fue realizado por un programador de tiempo.

Extracción: Primeramente se debía conocer cuando cosechar. Esto se realizaba con un pequeño disco blanco de plástico conectado perpendicularmente a un mango delgado (disco de Sechi). El momento de cosechar es oportuno cuando el disco ya no se ve a $2 \mathrm{~cm}$ de profundidad, manteniéndolo paralelo a la superficie del líquido.

En otoño-invierno: la cosecha se realizó cada dos o tres días.

En primavera-verano se cosechó diariamente.

En ambos períodos la extracción fue en forma manual: Alrededor de medio litro de pulpa por cada estanque. No se recomienda retirar más allá del $30 \%$ de las algas. Al término de la cosecha la pulpa se lavó con agua clorada para eliminar contaminación por gérmenes de cólera o hepatitis ( $\mathrm{pH} 5$, dos gotas de cloro en 1 litro de agua).

Filtrado: Inicialmente fue usado un tamizador vibratorio de área de tamizado 0,6x0,8 m, con tela fina de algodón. Posteriormente se emplearon tamices fijos, pero con un borde de $100 \mathrm{~mm}$ para retener más líquido, los que se dejaban escurrir sobre el estanque, apoyados sobre este. La recolección se realizó con el agitador detenido para dejar en reposo el cultivo y esperando que las algas se acercaran a la superficie.

Secado: Se empleó un secador solar de dos niveles de bandejas. Superficie de 1 x 0,8 m. Altura $0,3 \mathrm{~m}$. Construido en madera y aislado térmicamente con poliestireno expandido de $20 \mathrm{~mm}$ de espesor. La cubierta sobre el primer nivel de bandejas era de polietileno transparente y resistente a los rayos UV. Las bandejas eran dos marcos de listones recubiertos de tela fina de algodón. Un colector solar adicional de $1 \times 1 \mathrm{~m}$, cubierta de vidrio, con planchas de hierro pintadas de negro, estaba conectado a la zona inferior del secador. Tenía dos entradas de aire en la parte baja del colector y dos descargas en la superior del secador, en esta última empleando tubos de PVC de $100 \mathrm{~mm}$ de diámetro y $500 \mathrm{~mm}$ de longitud. El colector también era de madera y aislado con poliestireno. En otoño-invierno en dos días las algas estaban completamente secas. En primavera-verano bastaba un día. Las temperaturas máximas medidas en el secador fueron de 60 grados Celsius, en verano.

\section{Resultados y Discusión}

Las respuestas indicaron medias de producción diarias desde $3 \mathrm{~g} / \mathrm{m}^{2}$ en otoño-invierno hasta $4 \mathrm{~g} / \mathrm{m}^{2}$ en primavera-verano, con un costo de producción inferior a US\$7/kg, siendo la mitad de lo que cuesta la carne de res de la calidad más alta. En Chile se han calculado cifras de US\$13,5/kg en grandes instalaciones, pero allí deben emplearse equipos mayores, hay costes de consumo de energía de la red, transporte, gastos en personal, administración y mercadeo, entre otros. Se comercializa en farmacias a US\$ 200/kg en tabletas de 0,5 g. Vendido como polvo los precios a mayoristas se reducen casi a la mitad, unos US\$ 115/kg (datos de 2008).

\section{Conclusiones}

- El proceso productivo indicado es económico, fácil de implementar y mantener. Puede servir como un excelente complemento en la alimentación de una familia rural, pensando en $5 \mathrm{~m}^{2}$ de superficie cultivada por persona.

- Los precios de mercado son inalcanzables para una familia rural, sin embargo, pueden producir las microalgas para su consumo a un costo 16,4 veces inferior.

- Si se piensa en cultivos industriales, debe observarse a largo plazo la competencia externa: Algunos países orientales pueden ofrecer Spirulina en polvo a US\$ $6 / \mathrm{kg}$ precios de venta puesta en Chile (probablemente subsidiada), lo que tal vez generaría un desequilibrio en el mercado, sin embargo, no constituye una amenaza por ahora. Sus productos hasta hace poco, no eran aceptados en algunos países, debido a las restricciones sanitarias (se obtenía de complejos agrícolas donde el estiércol de los cerdos indirectamente estaba en la cadena productiva).

\section{Agradecimientos}

Al Proyecto Automatización del Proceso Productivo de Spirulina, DIPOG 8741-00, Universidad de Tarapacá, que financió la investigación y a los participantes del proyecto: Claudio Brieba, Ismelda Lobato, Delia Laime, Bernardo Barraza, Reinhold Schmidt, Leonardo Figueroa y Jaime Villanueva. 


\section{Bibliografía}

Belay, A.

2002 The potential application of Spirulina as a nutricional and therapeutic supplement in health management. JANA, 5: $27-48$

Earthrise Farms

1988 Five years testing of heavy metals in Spirulina: 1983-1987

Henrikson, R.

1994 Microalga Spirulina - Superalimento del futuro, Ed.Urano S.A., Barcelona, Spain.

Jourdan, J.P.

2002 Cultivez Votre Spiruline Manuel de Culture Artisanale de la Spiruline, Le Castanet, Mialet, 30140 Anduz, France. Pub. by Antenna Technologie,. email: jpj@bsi.fr.
Ponce, C. y Ponce, E.

2008 Design of Neuronal Network to Control Spirulina Aquaculture ICINCO-ICSO Press 2008: 289-292. ISBN 978-989-8111-30-2

Ponce, C.; Ponce, E. and Barraza, B.

2008 Measuring system for growth control of the Spirulina aquaculture. AIP Conference Proceedings, v. 1060, DOI: 10.1063/1.3037031, pp 113-116. The Smithsonian Institute/ NASA Astrophysics ISBN: 978-0-7354-0590-5 
